\title{
DESENVOLVIMENTO E VERIFICAÇÃO DE UM INSTRUMENTO DE AVALIAÇÃO DAS EXPECTATIVAS DE NOVOS USUÁRIOS DE APARELHOS DE AMPLIFICAÇÃO SONORA INDIVIDUAL
}

\author{
Development and verification of an instrument for \\ assessing expectations of new hearing aid users
}

\author{
Larissa Cristina Schuster ${ }^{(1)}$, Maristela Julio Costa ${ }^{(2)}$, Karine Thais Becker ${ }^{(3)}$, Tais Regina Hennig ${ }^{(4)}$
}

\begin{abstract}
RESUMO
Objetivo: desenvolver um instrumento para a mensuração da expectativa quanto ao uso de aparelho de amplificação sonora individual do paciente adulto/idoso atendido no SUS; e verificar o nível de confiabilidade para reprodutibilidade do mesmo. Método: participaram deste estudo 19 pacientes que aguardavam o recebimento de aparelho de amplificação sonora individual, através do Programa de Concessão de Próteses Auditivas de fluxo contínuo da Secretaria de Assistência à Saúde do Ministério da Saúde. O questionário contendo 12 questões, divididas em duas dimensões, foi aplicado utilizando-se a estratégia de teste e re-teste, com intervalo de sete dias entre a primeira e a segunda aplicação. Os dados obtidos foram submetidos à análise estatística para estimar o nível de concordância a partir do coeficiente Kappa simples. Resultados: a partir do levantamento dos relatos dos pacientes, colocados em forma de pergunta, obtivemos um pré-questionário. Este foi reformulado diversas vezes, à medida que se davam as aplicações piloto em grupos de pacientes voluntários, resultando na versão final apresentada. Os resultados obtidos no estudo de reprodutibilidade mostraram um nível de concordância satisfatório $(>0,61)$ para 9 questões $(75 \%)$, discreto $(0,41$ a 0,60$)$ para 2 questões $(16,6 \%)$ e fraco $(0,11$ a 0,40$)$ para apenas 1 questão $(8,3 \%)$. Conclusão: o instrumento testado apresenta um nível de reprodutibilidade satisfatório, sendo uma boa opção para avaliar a expectativa do paciente candidato ao uso de aparelho de amplificação sonora individual. Este instrumento pode ser útil, também, para nortear o trabalho de aconselhamento do paciente para o processo de seleção e adaptação de aparelho de amplificação sonora individual.
\end{abstract}

DESCRITORES: Questionários; Reabilitação de Deficientes Auditivos; Adulto; Idoso

(1) Fonoaudióloga do Projeto de Promoção da Saúde Auditiva da Universidade Federal de Santa Maria, UFSM, Santa Maria, RS; Aluna de mestrado do Programa de Pós-graduação em Distúrbios da Comunicação Humana na Universidade Federal de Santa Maria, UFSM, Santa Maria, RS.

(2) Fonoaudióloga; Professora Adjunta do Curso de Fonoaudiologia e do Programa de Pós-Graduação em Distúrbios da Comunicação Humana da Universidade Federal de Santa Maria, UFSM, Santa Maria, RS; Doutora em Ciências dos Distúrbios da Comunicação Humana pela Universidade Federal de São Paulo.

(3) Fonoaudióloga do Projeto de Promoção da Saúde Auditiva da Universidade Federal de Santa Maria, UFSM, Santa Maria, RS; Aluna de mestrado do Programa de Pós-graduação em Distúrbios da Comunicação Humana na Universidade Federal de Santa Maria, UFSM, Santa Maria, RS.

(4) Fonoaudióloga do Projeto de Promoção da Saúde Auditiva da Universidade Federal de Santa Maria, UFSM, Santa Maria, RS; Aluna de mestrado do Programa de Pós-graduação em Distúrbios da Comunicação Humana na Universidade Federal de Santa Maria, UFSM, Santa Maria, RS.

Conflito de interesses: inexistente

\section{INTRODUÇÃO}

A perda auditiva é assunto de crescente destaque na medida em que campanhas de conscientização e atenção à saúde auditiva ganham força na sociedade atual. Dessa forma, a procura por auxílio frente às dificuldades ocasionadas pela perda auditiva crescem a cada dia, principalmente entre a população adulta e idosa.

Em casos em que não há outra forma de tratamento, como o cirúrgico ou o medicamentoso, a opção mais adequada, quando indicada pelo médico otorrinolaringologista, é a adaptação de aparelho de amplificação sonora individual.

Atualmente, a tecnologia avançada na área audiológica possibilita inúmeras formas de amenizar as dificuldades enfrentadas por pacientes 
com perda auditiva, gerando, com frequência, expectativas muito elevadas com relação ao uso de aparelho de amplificação sonora individual. A expectativa sobre os aparelho de amplificação sonora individual parece ser o fator mais decisivo para uma adaptação bem sucedida ${ }^{1}$ e deve ser abordada antes da adaptação dos aparelhos de amplificação sonora individual, constituindo-se como parte da reabilitação auditiva² .

Considerando que a preocupação com o bemestar do paciente deve nortear as decisões do audiologista, é de fundamental importância investigar o que o paciente espera do seu processo de reabilitação auditiva a fim de melhor orientá-lo no decorrer de sua adaptação.

Para avaliação de aspectos subjetivos como a expectativa, estão disponíveis na literatura, alguns estudos que utilizaram questionários ou escalas para qualificar e quantificar as expectativas préadaptação dos indivíduos candidatos ao uso de aparelho de amplificação sonora individual ${ }^{2-3}$. O primeiro estudo avaliou 60 indivíduos adultos e idosos coma a aplicação do "The Expected Consequences of Hearing Aid Outcome" - $\mathrm{ECHO}^{4}$. O segundo, estudo brasileiro, avaliou amostra semeIhante ao presente trabalho, também aplicando um modelo de questionário próprio, desenvolvido especialmente para o estudo. Ressalta-se que o instrumento não se encontra disponível na literatura. É importante salientar que, apesar de sua existência, estes instrumentos, ou não estão publicados ou não se aplicam à realidade da população brasileira, usuária de aparelhos de amplificação sonora individual, principalmente quando esta, é a atendida pelo Sistema Único de Saúde - SUS.

Os propósitos deste estudo foram desenvolver um instrumento que possibilite a mensuração do grau de expectativa quanto ao uso de aparelho de amplificação sonora individual do paciente adulto/ idoso atendido no SUS; e verificar o nível de reprodutibilidade do mesmo.

\section{MÉTODO}

Participaram deste estudo 19 pacientes que aguardavam o recebimento de aparelho de amplificação sonora individual, através do Programa de Concessão de Próteses Auditivas de fluxo contínuo da Secretaria de Assistência à Saúde do Ministério da Saúde, desenvolvido em uma clínica-escola da Universidade Federal de Santa Maria. Foram excluídos da amostra os indivíduos com menos de 18 anos de idade, portadores de perda auditiva de grau severo ou profundo bilateral e aqueles que já tiveram algum tipo de experiência prévia com aparelho de amplificação sonora individual.
A construção do questionário baseou-se nos demais instrumentos relacionados ao tema disponíveis na literatura e, principalmente, nos relatos dos pacientes candidatos ao uso de aparelho de amplificação sonora individual, atendidos na clinica-escola onde se realizou o estudo.

Inicialmente realizou-se o levantamento dos principais relatos dos pacientes atendidos no serviço e colocando-os em forma de pergunta, resultando em uma lista de 26 questões. A partir daí, foram realizadas quatro aplicações-piloto, em pacientes voluntários que atendiam os critérios de inclusão deste estudo, tendo sido verificada a necessidade de adequações imediatas, que primeiramente foi reduzir ao máximo o número de questões do instrumento, com o objetivo de buscar uma versão viável, em termos de praticidade de aplicação e objetividade. Além disso, também realizou-se a substituição de alguns termos por outros que facilitassem a compreensão por parte do paciente e, principalmente, quanto a forma da resposta a ser dada.

A maior dificuldade encontrada foi especificamente quanto à escolha da melhor forma de resposta ao questionário, visto que necessitaríamos empregar uma forma simples e de fácil entendimento, para que não se perdesse o propósito desafiador de objetivar um aspecto totalmente subjetivo e ainda fazer com o que o instrumento resultante fosse prático e fácil de aplicar. Utilizamos para fins de teste, as escalas numéricas e também as respostas fechadas com necessidade de escolha de uma, dentre múltiplas possibilidades. À medida que eram realizadas as aplicações-piloto, percebemos que os pacientes compreendiam melhor e se posicionavam mais facilmente quanto à pergunta quando havia, no máximo, três possibilidades de resposta, dentre as quais ele deveria escolher apenas uma.

Após as adequações, a versão final do questionário ficou composta de 12 questões e será apresentada na exposição dos resultados, juntamente com a descrição sobre a forma de aplicação e interpretação do mesmo.

Para maior confiabilidade dos dados, a aplicação do questionário foi realizada sempre pelo mesmo profissional, sob as mesmas condições e sempre da mesma forma. Para verificação da reprodutibilidade do mesmo, utilizou-se a estratégia de teste e re-teste, com intervalo de sete dias entre a primeira e a segunda aplicação.

Para a realização deste estudo foi obtida a autorização formal dos indivíduos participantes para a utilização dos dados coletados, através da assinatura do "Termo de Consentimento Livre e Esclarecido". Ressalta-se que este estudo é vinculado ao projeto "Pesquisa e Base de Dados em Saúde 
Auditiva", registrado no Gabinete de Projetos sob o no 019731 e aprovado pelo Comitê de Ética em Pesquisa com certificado $n=0138.0 .243 .000-06$, em 05/12/2006.

Os dados obtidos foram organizados em um banco de dados, através do programa Excel e submetidos à análise estatística para estimar o nível de concordância a partir do coeficiente Kappa simples, utilizando-se o programa STATA, versão 10.0 Statistics/Data Analysis. Os valores de concordância considerados estão de acordo com os sugeridos pela literatura especializada ${ }^{5}$, onde $\mathrm{k}<0,10$ (ausência de concordância); de 0,11 a 0,40 (fraca); 0,41 a 0,60 (discreta); de 0,61 a 0,80 (moderada); de 0,81 a 0,99 (substancial) e 1,0 (concordância perfeita).

\section{RESULTADOS}

Será apresentado a seguir o questionário desenvolvido (figura 1), após os ajustes e considerações necessárias, com base no estudo de reprodutibilidade do instrumento.

O questionário contém 12 questões, divididas em duas dimensões. A primeira, constituída de sete questões, aborda as expectativas do paciente quanto ao uso dos aparelhos auditivos em situações familiares, com pessoas desconhecidas, em locais ruidosos, ao telefone, ao assistir televisão ou ouvir rádio, e a expectativa quanto ao restabelecimento de suas condições auditivas. Já a segunda, constituída de cinco questões, aborda as preocupações quanto ao uso da amplificação, incluindo aspectos diretamente relacionados ao manuseio dos aparelhos de amplificação sonora individual, aspectos emocionais, estéticos e a possível influência de terceiros no processo de adaptação.

$O$ instrumento traz como opções de respostas as alternativas NÃO (1 ponto), TALVEZ/NÃO SEI (2 pontos) e SIM (3 pontos). Dessa forma, quanto às expectativas, a pontuação mínima possível é de 7 pontos e representa o nível mínimo ou ausência de expectativas. Por outro lado, a pontuação máxima nesta mesma dimensão é de 21 pontos, a qual representa o nível máximo de expectativas do paciente quanto ao uso de aparelho de amplificação sonora individual. Já quanto às preocupações, a pontuação mínima possível é de 5 pontos e representa o mínimo de preocupação. A pontuação máxima possível é de 15 pontos, e retrata o nível máximo de preocupação do paciente quanto ao uso de aparelho de amplificação sonora individual.

O estudo, conforme já exposto, seguiu a estratégia teste-reteste, sendo que o questionário foi aplicado em um grupo de 19 sujeitos, constituído por sete mulheres e 12 homens, com idades entre
24 e 88 anos, candidatos ao uso de aparelho de amplificação sonora individual e que se adequaram aos critérios de exclusão acima descritos.

É importante ressaltar que foi verificada a necessidade de adequação de duas questões, diferenciando-as para aplicação em indivíduos adultos e idosos. Assim sendo, o questionário foi compilado e apresenta, simultaneamente, as questões direcionadas aos indivíduos adultos e idosos, novos usuários de aparelho de amplificação sonora individual. A diferenciação entre as questões dirigidas especificamente aos indivíduos idosos, ficou determinada pela indicação do asterisco $\left({ }^{*}\right)$.

Os resultados obtidos no estudo de reprodutibilidade do questionário mostraram, um nível de concordância satisfatório $(>0,61)$ para 9 questões $(75 \%)$, discreto $(0,41$ a 0,60$)$ para 2 questões $(16,6 \%)$ e fraco $(0,11$ a 0,40$)$ para apenas 1 questão (8,3\%), conforme a tabela 1 .

\section{DISCUSSÃO}

O desenvolvimento de um instrumento para avaliação das expectativas do paciente candidato ao uso de aparelhos de amplificação sonora individual foi uma iniciativa que visou suprir as necessidades da área da audiologia em avaliar objetivamente tais aspectos.

No processo de desenvolvimento do questionário, deparamo-nos com diversos impasses, os quais serão descritos na discussão à seguir.

Inicialmente, discutiremos a forma de apresentação do questionário. O formato das questões apresentadas em duas dimensões foi resultado de diversas tentativas anteriores, as quais nos permitiram observar, bem como em outros estudos ${ }^{6}$, que os questionários extensos e com questões muito específicas, dificultam a sua aplicação por parte do examinador, bem como a compreensão das questões por parte do individuo avaliado. $\mathrm{Da}$ mesma forma, os aspectos relacionados com a auto-percepção da dificuldade auditiva e aqueles relacionados com fatores extrínsecos ao individuo, mostraram-se melhor dimensionados e contextualizados, quando analisados separadamente.

Por exemplo, se o paciente possui um tipo de perda muito complexa para adaptação da prótese, as habilidades auditivas encontram-se muito alteradas, ou ainda, a idade do paciente é muito avançada e a sua expectativa é de que todos os seus problemas auditivos sejam resolvidos com a protetização, pode haver uma combinação de fatores que impeçam que tal expectativa seja alcançada. Dessa forma, sua satisfação é de difícil alcance, pois a expectativa com relação aos aspectos positivos do processo era muito alta. 


\section{QUESTIONÁRIO PARA AVALIAÇ̃̃O DAS EXPECTATIVAS DO ADULTO/IDOSO*, NOVO USUÁRIO DE APARELHO DE AMPLIFICAÇÃO SONORA INDIVIDUAL}

Instruções: Este questionário constitui-se em um instrumento que permite ao audiologista conhecer o que espera um paciente adulto/idoso, candidato ao uso de aparelho de amplificação sonora individual, com relação a sua reabilitação. O mesmo possui 12 questões, divididas em duas escalas ou dimensões, das quais uma é referente às expectativas e outra às preocupações destes pacientes, abordando os principais aspectos envolvidos nos momentos que antecedem a primeira experiência com aparelho de amplificação sonora individual. $\mathrm{O}$ instrumento deverá ser aplicado pelo profissional responsável, sendo que todas as questões devem ser lidas e explicadas ao paciente, sempre que necessário. Para todas as questões é possível apenas uma opção de resposta.

\section{Expectativas}

Com o uso dos aparelhos de amplificação sonora individual, $o(a) \operatorname{Sr}(a)$ :

1. Espera entender mais facilmente as pessoas com quem convive?
( ) NÃO
( ) TALVEZ/NÃO SEI
( ) SIM

2. Espera que a convivência com a sua família, amigos ou colegas de trabalho melhore, pois vai conseguir compreendê-los mais facilmente?

* Espera que a convivência com a sua família e amigos melhore, pois vai conseguir compreendê-los mais facilmente?
( ) NÃO
( ) TALVEZ/NÃO SEI
( ) SIM

3. Espera sentir-se mais a vontade para conversar com estranhos e freqüentar estabelecimentos comerciais e de serviços em geral?

* Espera sentir-se mais a vontade para conversar com estranhos e/ou mais independente para freqüentar estabelecimentos comerciais e de serviços em geral, sem acompanhante?
( ) NÃO
( ) TALVEZ/NÃO SEI
( ) SIM

4. Espera entender melhor as pessoas em locais barulhentos como reuniões, restaurantes, festas, cultos religiosos?
( ) NÃO
( ) TALVEZ/NÃO SEI
( ) SIM

5. Espera entender melhor ao telefone?
( ) NÃO
( ) TALVEZ/NÃO SEI
( ) SIM

6. Espera entender melhor a TV ou rádio com o volume mais baixo?
( ) NÃO
( ) TALVEZ/NÃO SEI
( ) SIM

7. Espera escutar como escutava antes de ter problema de audição?
( ) NÃO
( ) TALVEZ/NÃO SEI
( ) SIM

\section{Preocupações}

Quando for um usuário de prótese(s) auditiva(s), o(a) $\operatorname{Sr}(a)$ :

8. Os cuidados com a(s) prótese(s) auditiva(s) serão motivo de preocupação (saber/lembrar de colocá-las, trocar a pilha, observar os cuidados quanto à higiene, etc)?
( ) NÃO
( ) TALVEZ/NÃO SEI
( ) SIM

9. Pensa que a(s) prótese(s) auditiva(s) farão você parecer uma pessoa deficiente, velha e/ou incapaz?
( ) NÃO
( ) TALVEZ/NÃO SEI
( ) SIM

10. Do ponto de vista estético, vai se sentir desconfortável/incomodado usando a(s) prótese(s) auditiva(s)?
( ) NÃO
( ) TALVEZ/NÃO SEI
( ) SIM

11. Espera que o uso da(s) prótese(s) auditiva(s) seja temporário e que, através do uso sua audição pode vir a melhorar, até não precisar mais delas?
( ) NÃO
( ) TALVEZ/NÃO SEI
( ) SIM

12. Tem alguma experiência prévia de insucesso ou relato de algum conhecido ou familiar que não teve bom resultado com o uso de aparelho de amplificação sonora individual?
( ) NÃO
( ) TALVEZ/NÃO SEI
( ) SIM

\section{Figura 1}




\begin{tabular}{cccc}
\hline Questão & $\begin{array}{c}\text { Porcentagem de } \\
\text { concordância } \\
\text { teste/reteste (\%) }\end{array}$ & Coeficiente Kappa (k) & Concordância \\
\hline $\mathbf{1}$ & 94,7 & 0,88 & Substancial \\
$\mathbf{2}$ & 100 & 1 & Perfeita \\
$\mathbf{3}$ & 100 & 1 & Perfeita \\
$\mathbf{4}$ & 94,7 & 0,88 & Substancial \\
$\mathbf{5}$ & 94,7 & 0,88 & Substancial \\
$\mathbf{6}$ & 100 & 1 & Perfeita \\
$\mathbf{7}$ & 52,6 & 0,28 & Fraca \\
$\mathbf{8}$ & 78,9 & 0,78 & Moderada \\
$\mathbf{9}$ & 89,5 & 0,84 & Substancial \\
$\mathbf{1 0}$ & 63,2 & 0,44 & Discreta \\
$\mathbf{1 1}$ & 63,2 & 0,44 & Discreta \\
$\mathbf{1 2}$ & 73,7 & 0,75 & Moderada \\
\hline
\end{tabular}

Análise realizada utilizando-se o coeficiente Kappa simples, onde $k<0,10$ (ausência de concordância); de 0,11 a 0,40 (fraca); 0,41 a 0,60 (discreta); de 0,61 a 0,80 (moderada); de 0,81 a 0,99 (substancial) e 1,0 (concordância perfeita).

Por outro lado, o paciente que chega à clínica com conceitos pré-concebidos relacionados ao aparelho de amplificação sonora individual, pode dificultar sua própria aceitação do processo de adaptação, e assim influenciar a obtenção de bons resultados e, consequentemente a satisfação. A influência de usuários que estão próximos ao paciente ou até mesmo da mídia poderá fazer com que o paciente tenha mais dificuldade em colaborar e perceber o quanto os aparelhos de amplificação sonora individual estão the proporcionando melhoras em seu processo comunicativo.

O contrário também pode se tornar verdadeiro, em uma situação em que o paciente, mesmo sendo resistente ao uso de aparelhos de amplificação sonora individual por conhecer casos de insucesso, acaba tendo suas expectativas superadas a partir da experiência auditiva com o uso de amplificação.

Assim sendo, verificamos que as sete questões que envolvem as expectativas e as cinco questões que envolvem os aspectos negativos da protetização, contemplam satisfatoriamente os aspectos mais freqüentes e relevantes observados na rotina clinica, pois levantam de forma objetiva o que estamos investigando, além de não ser muito extenso, facilitando assim a aplicação, pois não demanda muito tempo e nem cansa o paciente.

Considerando ainda a forma de resposta fechada, acreditamos que, para tratar de um aspecto tão subjetivo quanto a expectativa, o ideal seria utilizar um material que não oferecesse risco de direcionar o indivíduo a algum tipo conhecido de resposta, como ocorre nos questionários que utilizam este tipo de respostas. No entanto, a opção final por uma forma estruturada, onde o tipo de resposta é fechado torna a aplicação do instrumento mais objetiva e rápida, bem como a análise das respostas obtidas ${ }^{6}$, principalmente quando se trata do contexto de pesquisa.

Além disso, a opção por três alternativas de resposta (NÃO; NÃO SEI/TALVEZ; SIM), objetivou possibilitar também a neutralidade às respostas dos indivíduos avaliados. A ausência da categoria central NÃO SEI/TALVEZ, poderia conduzir o entrevistado a uma tendência positiva ou negativa irreal ${ }^{7}$.

A escolha do tipo de questão deve estar de acordo também com as características da população em estudo ${ }^{6}$. Dessa forma, a escolha de três categorias de resposta também se deve ao fato de que percebemos, ao longo do estudo, que escalas de graduação com muitas alternativas dificultam o posicionamento do individuo avaliado, principalmente, quando tratam-se de pacientes idosos.

Outro aspecto que se constitui um desafio quando se trata da elaboração de um instrumento, é a difícil tarefa de fazer com que as questões fiquem totalmente claras e acessíveis a população que se deseja estudar. Em nosso estudo, verificamos a necessidade do emprego de uma linguagem menos científica e mais simplificada ou, em outros casos, a reformulação total da forma de abordar a questão.

Por sua vez, com relação à reprodutibildade, observou-se que o questionário avaliado apresentou alto nível de concordância na maioria das questões que o compõe (75\%). Aquelas que não apresentaram um nível de concordância aceitável 
(questões 7, 10 e 11), obtiveram níveis fracos ou discretos de concordância.

Determinar o nível de concordância de um instrumento é de fundamental importância, pois associada a este está a qualidade dos dados que serão coletados através dele ${ }^{8,9}$.

Segundo a literatura ${ }^{10}$, é desejável, para os estudos exploratórios, um nível de concordância superior a 0,61 (satisfatória), no entanto niveis entre 0,50-0,60 são também considerados. Ainda, segundo o autor, devem ser eliminadas apenas as questões com níveis de reprodutibilidade inferior à 0,20 ou aquelas com efeito negativo. Estudos semeIhantes utilizaram os mesmos parâmetros indicados pelo autor ${ }^{11-15}$.

O valor mínimo de concordância encontrado no estudo foi 0,28 (fraca), não sendo, portanto, obrigatória a sua eliminação.

Acredita-se que a maior variação de respostas nas questões apontadas se relaciona ao fato destas tratarem de experiências novas e envolverem aspectos nunca antes pensados pelos pacientes. Dessa forma, julgamos natural que houvesse variação das respostas, tendo em vista que o paciente poderia estar em processo de formação de opinião sobre o assunto. Devido à relevância das questões, optou-se pela manutenção das mesmas no questionário, mediante alguns ajustes.

Uma das três questões que apresentaram menor nível de reprodutibilidade, pertence ao conjunto de variáveis relacionadas às expectativas: " 7 - Espera escutar como escutava antes de ter problema de audição?". Esta trata dos resultados que o paciente espera alcançar com o uso dos aparelhos de amplificação sonora individual, comparado à audição que um dia tivera. Observamos que com a adequação da questão para: "7 - Espera escutar como escutava quando não percebia a dificuldade auditiva?", tornou-se mais claro o objetivo da pergunta. No caso dos indivíduos idosos o formato "7 - Espera escutar como escutava quando era mais jovem?" se tornou ainda mais claro.

O mesmo ocorreu nas outras duas questões que apresentaram baixo nível de concordância, as quais pertencem ao conjunto de variáveis relacionadas às preocupações: "10 - Do ponto de vista estético, vai se sentir desconfortável/incomodado usando os aparelhos de amplificação sonora individual?" e "11 - Espera que o uso dos aparelhos de amplificação sonora individual seja temporário e que, através do uso, sua audição pode vir a melhorar até não precisar mais delas?". Verificamos que os seguintes ajustes, tornaram mais claros também os objetivos destas questões: "10 - Do ponto de vista estético, vai se sentir pouco à vontade/envergonhado usando os aparelhos de amplificação sonora individual?" e "11 - Espera que os aparelhos de amplificação sonora individual funcionem como um 'remédio', o qual será utilizado temporariamente e que vai 'curar' a audição?".

Finalmente percebemos que é muito importante a preocupação com que o paciente pensa e sente no momento de decidir sobre o uso de aparelhos de amplificação sonora individual. Por isso ressaltamos a importância do desenvolvimento de trabaIhos como este, que visam disponibilizar instrumentos que venham a auxiliar tanto o fonoaudiólogo como o paciente, contribuindo para um satisfatório processo de reabilitação auditiva.

\section{CONCLUSÃO}

Os resultados encontrados sugerem que o instrumento testado apresenta um nível de reprodutibilidade satisfatório, sendo uma boa opção para avaliar o nível de expectativa do paciente candidato ao uso de aparelhos de amplificação sonora individual. Este instrumento pode ser útil, também, para nortear o trabalho de aconselhamento e preparação do paciente dentro do processo de seleção e adaptação de aparelhos de amplificação sonora individual.

\section{AGRADECIMENTOS}

À Fga. Sinéia Neujahr dos Santos, pelo valioso auxílio prestado na análise estatística dos dados obtidos neste estudo. 


\begin{abstract}
Purpose: to develop an instrument to measure the expectative degree regarding the use of hearing aids for an adult/elderly patient referred at SUS; and check for its reproducibility level. Method: 19 patients awaiting hearing aids, provided from the Ministry of Heath, thought the Grant Hearing Aids Program of the Secretary of Health Care from the Ministry of Health were submitted to a questionnaire. The questionnaire contained 12 questions, divided in two dimensions, was applied using the strategy of test and re-test, with an interval of seven days between the first and the second application. Data were submitted to a statistical analysis in order to estimate the level of agreement using the simple Kappa coefficient. Results: from a survey of patient reports, as a question, we obtained a prequestionnaire. It has been redrafted several times, as they gave the pilot applications in group of volunteer patients, resulting in the final version. The obtained results showed a satisfactory level of agreement $(>0.61)$ for nine questions $(75 \%)$, mild $(0.41$ to 0.60$)$ for two questions $(16.6 \%)$ and low ( 0.11 to 040$)$ to only one question $(8.3 \%)$. Conclusion: the tested instrument has a satisfactory level reproducibility and is a good option in order to evaluate the patient's expectation level when being candidate for using the new hearing aids. This instrument can also be useful to guide the work of counseling and patient preparation in the selection and fitting process of the hearing aids.
\end{abstract}

KEYWORDS: Questionnaires; Hearing Aids; Rehabilitation of Hearing Impairment; Adult; Aged

\section{REFERÊNCIAS}

1. Suman P, Blasca WQ, Ferrari DV. Avaliação subjetiva da expectativa quanto ao uso do aparelho de amplificação sonora individual: correlação com aspectos auditivos, sociais e econômicos. Distúrb Comun. 2008; 20(1): 107-14.

2. Saunders GH, Lewis MS, Forsline A. Expectations, prefitting counseling, and hearing aid outcome. J Am Acad Audiol; 2009; 20(5): 320-34.

3. Santos I, Couto CM. Expectativa e Satisfação do Idoso Em Relação à Prótese Auditiva. 16o Congresso Brasileiro de Fonoaudiologia; 24 a 27 de set. Campos do Jordão, SP, Brasil; 2008.

4. Cox RM, Alexander GC. Expectations about hearing aids and their relationship to fitting outcome. J Am Acad Audiol. 2000;11(7):368-80.

5. Shout PE. Measurement reliability and agreement in psychiatry. Stat Methods Med Res. 1998; 7(3): 301-17.

6. Amaro A, Póvoa A, Macedo I. A arte de fazer questionários. [Trabalho de conclusão de disciplina]. Universidade do Porto; Cidade do Porto; 2005.

7. Alexandre JWC, Andrade DF de, Vasconcelos AP de, Araújo MAS de, Batista MJ. Análise do número de categorias da escala de Likert aplicada à gestão pela qualidade total através da teoria da resposta ao item. XXIII Encontro Nac. de Eng. de Produção; 21 a 24 de out. Ouro Preto, MG, Brasil; 2003.
8. Farias JrJC, PiresMC, LopesAS. Reprodutibilidade de um questionário para o levantamento de informações sobre comportamentos relacionados à saúde em adolescentes. Rev. Bras. Ciência e Mov. 2002; 10: 43-8.

9. Campos MR, Leal MC, Souza Jr PR de, Cunha $\mathrm{CB}$ da. Consistência entre fontes de dados e confiabilidade interobservador do Estudo da Morbimortalidade e Atenção Peri e Neonatal no Município do Rio de Janeiro. Cad. Saúde Pública. 2004; Sup 1:S34-43.

10. Ravid R. Practical statistics for educators. University Press of America, New York, 2000.

11. Leite IHM, Waissmann W, Veggi AB. Desenvolvimento e reprodutibilidade de questionário para avaliar práticas e conhecimentos em segurança alimentar de nutricionistas da área clínica. Rev. Nutr. 2007; 20(4): 397-404.

12. Nahas MV, Rabacow FM, Pereira SV, Borgatto AF. Reprodutibilidade de uma escala para avaliar a percepção dos trabalhadores quanto ao ambiente e às condições de trabalho. Rev. Bras. Saúde Ocup. 2009; 34 (120): 179-83.

13. Camara VM, Souza DPO de, Filhote MIF, Luiz RR, Souza CO de, Meyer A. Confiabilidade de questionário de conhecimento sobre exposição ao mercúrio na produção de ouro. Rev. Saúde Pública. 2009; 43(2): 373-6. 
14. Lima TC de. Elaboração, validação e aplicação de um instrumento para caracterização de uma população com 50 anos ou mais portadora do HIV/ AIDS. [Dissertação de mestrado]. Universidade Estadual de Campinas - UNICAMP - Faculdade de Ciências Médicas, Campinas, SP, 2009.
15. Zanolla AF, Olinto MTA, Henn RL, Wahrlich V., Anjos LA dos. Avaliação de reprodutibilidade e validade de um questionário de freqüência alimentar em adultos residentes em Porto Alegre, Rio Grande do Sul, Brasil. Cad. Saúde Pública. 2009; 25(4): 840-8.

http://dx.doi.org/10.1590/S1516-18462011005000069

RECEBIDO EM: 20/08/2010

ACEITO EM: 24/03/2011

Endereço para correspondência:

Larissa Cristina Schuster

Rua Duque de Caxias, 1505/308 - Centro

Santa Maria - RS

CEP: $97015-190$

E-mail: larissa.schuster@gmail.com

Rev. CEFAC. 2012 Mar-Abr; 14(2):215-222 